\title{
Macrolide therapy in airways disease
}

\section{Le traitement par macrolides en cas de maladie des voies aériennes}

$I^{n}$ $\mathrm{n}$ the present issue of the Canadian Respiratory Journal, Porhownik et al (1) report inconclusive results of a small trial of continuous macrolide antibiotic therapy in post-transplant patients with bronchiolitis obliterans syndrome. This report typifies an increasing interest in chronic macrolide therapy in a wide variety of airway diseases.

The initial enthusiasm for macrolide therapy was generated by its beneficial effect in diffuse panbronchiolitis, a disease described by and largely limited to the Japanese (2). Relatively low-dose chronic erythromycin treatment is the therapy of choice in this disease, and apparently has had a

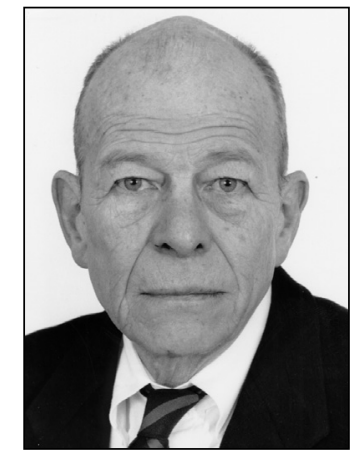

Nick R Anthonisen ans le présent numéro du Journal canadien de Uespirologie, Porhownik et coll. (1) communiquent les résultats non concluants d'un petit essai sur une antibiothérapie continue par macrolides chez des patients greffés atteints du syndrome de bronchiolite oblitérante. Ce rapport illustre l'intérêt croissant envers le traitement par macrolides chroniques pour une vaste gamme de maladies des voies aériennes.

L'enthousiasme envers le traitement par macrolides provient de son effet bénéfique sur la panbronchiolite diffuse, une maladie décrite par les Japonais et qui se limite en grande partie à cette major effect on improving lung function and possibly survival (3). The dose of erythromycin used is less than that used for infections, and the effect of the drug has been ascribed to antiinflammatory properties of the drug, shown in numerous in vitro studies $(4,5)$.

Although diffuse panbronchiolitis appears to have a specific pathology, it is an inflammatory disease of the small airways, with signs and symptoms of progressive airways obstruction. It seemed reasonable, therefore, to try macrolide therapy in other airway diseases. A small trial in patients with bronchiectasis showed improved lung function and reduced sputum (6), but this could have been due to the antimicrobial action of the macrolide antibiotic. Beneficial results have been reported in asthma (7) and the positive results have been ascribed to putative antibiotic effects; however, I am unaware of randomized trials with macrolide therapy in asthma.

More promising results have emerged in cystic fibrosis. In a number of small trials (8-10), macrolide therapy has been associated with improvements in lung function and quality of life, weight maintenance and reductions in exacerbations. The drug used was usually azithromycin, and the doses used were in the range between 'low' and 'therapeutic' in terms of antibacterial action. It is likely that macrolides are now commonly used in cystic fibrosis, although it might be fair to say that it is not clear how much they contribute to patient health, ie, results are probably less impressive than in diffuse panbronchiolitis. Changes in sputum suggestive of anti-inflammatory effects have not been observed in cystic fibrosis (11).

There have been few trials of chronic macrolide therapy in chronic obstructive pulmonary disease, and those that have been published do not show promising results (12). A chronic obstructive pulmonary disease research network, funded by the National Institutes of Health, is currently examining this issue, with exacerbation frequency as the end point. It is an enormous trial, with a projected total of 1000 patients who have severe disease. Definitive results from the study should emerge in approximately two years.

The study by Porhownik et al (1), using macrolides in posttransplant patients with bronchiolitis obliterans was stimulated population (2). Des doses relativement faibles d'un traitement par érythromycine chronique représentent le traitement de choix contre cette maladie et ont apparemment eu un effet considérable sur l'amélioration de la fonction pulmonaire et peut-être la survie (3). La dose d'érythromycine utilisée est inférieure à celle utilisée contre les infections, et son effet est attribué à ses propriétés anti-inflammatoires, démontrées dans de nombreuses études in vitro $(4,5)$.

Bien que la panbronchiolite diffuse semble dotée d'une pathologie précise, c'est une maladie inflammatoire des petites voies aériennes, aux signes et symptômes d'occlusion évolutive des voies aériennes. Il semble donc raisonnable d'essayer un traitement par macrolides dans d'autres maladies des voies aériennes. Un petit essai chez des patients atteints de bronchiectasie a démontré une amélioration de la fonction pulmonaire et une diminution des expectorations (6), qui pourrait être causée par l'action antimicrobienne de l'antibiotique macrolide. On a déclaré des effets bénéfiques dans l'asthme (7), et les résultats positifs ont été attribués à de prétendus effets antibiotiques. Je ne connais toutefois aucun essai aléatoire sur les traitements aux macrolides contre l'asthme.

Des résultats plus prometteurs s'observent dans le cas de la fibrose kystique. Dans plusieurs petits essais (8-10), le traitement par macrolides s'associe à des améliorations de la fonction pulmonaire et de la qualité de vie, au maintien du poids et à la réduction des exacerbations. En général, on utilisait de l'azithromycine, et les doses variaient entre «faibles » et «thérapeutiques » pour ce qui est de leur action antibactérienne. Il est probable que l'usage des macrolides soit maintenant généralisé en cas de fibrose kystique, mais il serait honnête d'affirmer qu'on ne sait pas à quel point les macrolides peuvent contribuer à la santé des patients, c'est-à-dire que les résultats sont probablement moins impressionnants que dans le cas de la panbronchiolite diffuse. Dans la fibrose kystique, on n'a pas observé de changements des expectorations évocateurs d'un effet anti-inflammatoires (11).

Quelques essais ont porté sur la thérapie par macrolides chroniques en cas de maladie pulmonaire obstructive chronique, et ceux qui ont été publiés ne donnent pas des 
by previous findings that found macrolide therapy was associated with improvements in lung function in patients with this extremely difficult and discouraging complication of lung transplantation. If macrolides are indeed associated with improvements in bronchiolitis obliterans, then it is an important treatment option. On the other hand, it should be stressed that these are preliminary findings. Porhownik et al (1) found that while macrolides were associated with meaningful improvements in lung function in two patients, this was not the case for the group as a whole. In addition, there was no placebo-treated control group. It seems as suggested by Porhwnik et al (1) that a randomized trial will be necessary to establish macrolides as a useful therapy of bronchiolitis obliterans.

Nick R Anthonisen MD Editor-in-Chief, Canadian Respiratory Journal

\section{REFERENCES}

1. Porhownik NR, Batobara W, Kepron W, Unruh HW, Bshouty Z. Effect of maintenance azithromycin on established bronchiolitis obliterans syndrome in lung transplant patients. Can Respir J 2008;15:199-202.

2. Koyama H, Geddes DM. Erythromycin and diffuse panbronchiolitis. Thorax 1997;52:915-8.

3. Kudoh S, Azuma A, Yamamoto M, Izumi T, Ando M. Improvement of survival in patients with diffuse panbronchiolitis treated with low-dose erythromycin. Am J Respir Crit Care Med 1998;157:1829-32.

4. Vanaudenaerde BM, Wuyts WA, Geudens N, et al. Macrolides inhibit IL17-induced IL8 and 8-isoprostane release from human airway smooth muscle cells. Am J Transplant 2007;7:76-82.

5. Takizawa H, Desaki M, Ohtoshi T, et al. Erythromycin modulates IL-8 expression in normal and inflamed human bronchial epithelial cells. Am J Respir Crit Care Med 1997;156:266-71.

6. Tsang KW, Ho PI, Chan KN, et al. A pilot study of low-dose erythromycin in bronchiectasis. Eur Respir J 1999;13:361-4.

7. Kraft M, Cassell GH, Pak J, Martin RJ. Mycoplasma pneumoniae and Chlamydia pneumoniae in asthma: Effect of clarithromycin. Chest 2002;121:1782-8.

8. Saiman L, Marshall BC, Mayer-Hamblett N, et al. Azithromycin in patients with cystic fibrosis chronically infected with Pseudomonas aeruginosa: A randomized controlled trial. JAMA 2003;290:1749-56.

9. Wolter J, Seeney S, Bell S, Bowler S, Masel P, McCormack J. Effect of long term treatment with azithromycin on disease parameters in cystic fibrosis: A randomised trial. Thorax 2002;57:212-6.

10. Clement A, Tamalet A, Leroux E, Ravilly S, Fauroux B, Jais JP. Long term effects of azithromycin in patients with cystic fibrosis: A double blind, placebo controlled trial. Thorax 2006;61:895-902.

11. Equi A, Balfour-Lynn IM, Bush A, Rosenthal M. Long term azithromycin in children with cystic fibrosis: A randomised, placebo-controlled crossover trial. Lancet 2002;360:978-84.

12. Banerjee D, Honeybourne D, Khair OA. The effect of oral clarithromycin on bronchial airway inflammation in moderate-tosevere stable COPD: A randomized controlled trial. Treat Respir Med 2004;3:59-65. résultats prometteurs (12). Un réseau de recherche sur les maladies pulmonaires obstructives chroniques, fondé par les National Institutes of Health, porte sur cette question, la fréquence des exacerbations représentant le paramètre ultime. C'est un énorme essai, au total projeté de 1000 patients atteints d'une maladie grave. Les résultats définitifs de l'étude devraient être publiés d'ici environ deux ans.

L'étude de Porhownik et coll. (1), faisant appel aux macrolides chez des patients greffés atteints de bronchiolite oblitérante, découle d'observations antérieures selon lesquelles le traitement par macrolides s'associait à des améliorations de la fonction pulmonaire chez des patients atteints de cette complication de la greffe pulmonaire extrêmement complexe et décourageante. Si les macrolides s'associent vraiment à une amélioration de la bronchiolite oblitérante, c'est une importante possibilité de traitement, Par contre, il faut souligner qu'il s'agit de résultats préliminaires. Porhownik et coll. (1) ont constaté que si les macrolides s'associaient à une amélioration significative de la fonction pulmonaire chez deux patients, ce n'était pas le cas dans l'ensemble du groupe. De plus, il n'y avait pas de groupe témoin traité par placebo. Comme l'ont suggéré Porhownik et coll. (1), il semble qu'un essai aléatoire s'impose pour établir si les macrolides représentent un traitement utile de la bronchiolite oblitérante.

Nick R Anthonisen MD Rédacteur en chef, Journal canadien de respirologie

\section{RÉFÉRENCES}

1. Porhownik NR, Batobara W, Kepron W, Unruh HW, Bshouty Z. Effect of maintenance azithromycin on established bronchiolitis obliterans syndrome in lung transplant patients. Can Respir J 2008;15:199-202.

2. Koyama H, Geddes DM. Erythromycin and diffuse panbronchiolitis. Thorax 1997;52:915-8.

3. Kudoh S, Azuma A, Yamamoto M, Izumi T, Ando M. Improvement of survival in patients with diffuse panbronchiolitis treated with low-dose erythromycin. Am J Respir Crit Care Med 1998;157:1829-32.

4. Vanaudenaerde BM, Wuyts WA, Geudens N et coll. Macrolides inhibit IL17-induced IL8 and 8-isoprostane release from human airway smooth muscle cells. Am J Transplant 2007;7:76-82.

5. Takizawa H, Desaki M, Ohtoshi T et coll. Erythromycin modulates IL-8 expression in normal and inflamed human bronchial epithelial cells. Am J Respir Crit Care Med 1997;156:266-71.

6. Tsang KW, Ho PI, Chan KN et coll. A pilot study of low-dose erythromycin in bronchiectasis. Eur Respir J 1999;13:361-4.

7. Kraft M, Cassell GH, Pak J, Martin RJ. Mycoplasma pneumoniae and Chlamydia pneumoniae in asthma: Effect of clarithromycin. Chest 2002;121:1782-8.

8. Saiman L, Marshall BC, Mayer-Hamblett N et coll. Azithromycin in patients with cystic fibrosis chronically infected with Pseudomonas aeruginosa: A randomized controlled trial. JAMA 2003;290:1749-56.

9. Wolter J, Seeney S, Bell S, Bowler S, Masel P, McCormack J. Effect of long term treatment with azithromycin on disease parameters in cystic fibrosis: A randomised trial. Thorax 2002;57:212-6.

10. Clement A, Tamalet A, Leroux E, Ravilly S, Fauroux B, Jais JP. Long term effects of azithromycin in patients with cystic fibrosis: A double blind, placebo controlled trial. Thorax 2006;61:895-902.

11. Equi A, Balfour-Lynn IM, Bush A, Rosenthal M. Long term azithromycin in children with cystic fibrosis: A randomised, placebo-controlled crossover trial. Lancet 2002;360:978-84.

12. Banerjee D, Honeybourne D, Khair OA. The effect of oral clarithromycin on bronchial airway inflammation in moderate-tosevere stable COPD: A randomized controlled trial. Treat Respir Med 2004;3:59-65. 


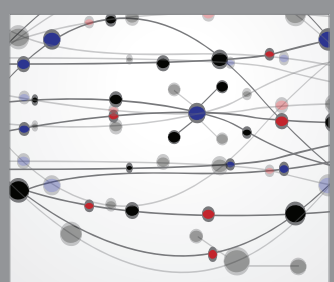

The Scientific World Journal
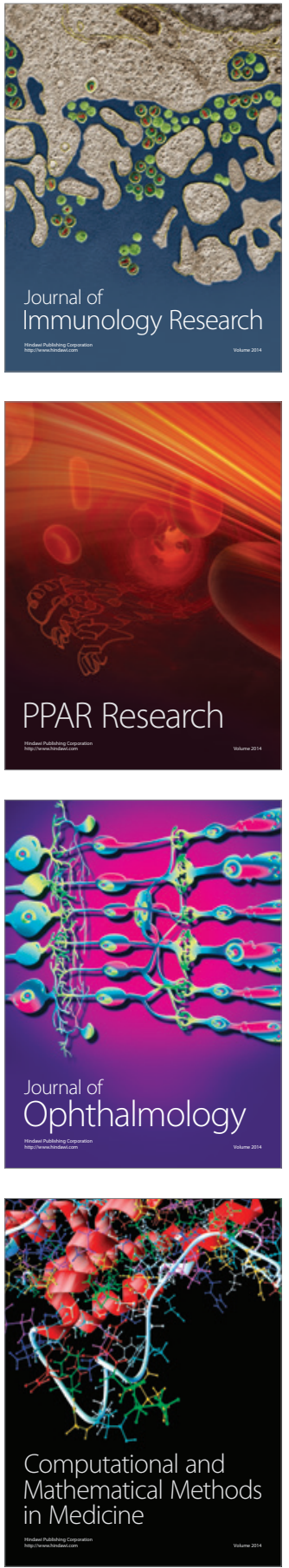

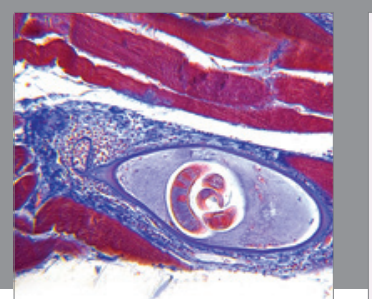

Gastroenterology Research and Practice

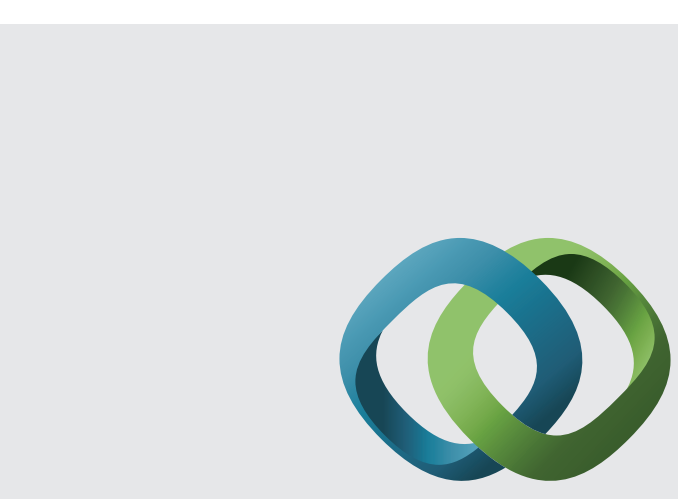

\section{Hindawi}

Submit your manuscripts at

http://www.hindawi.com
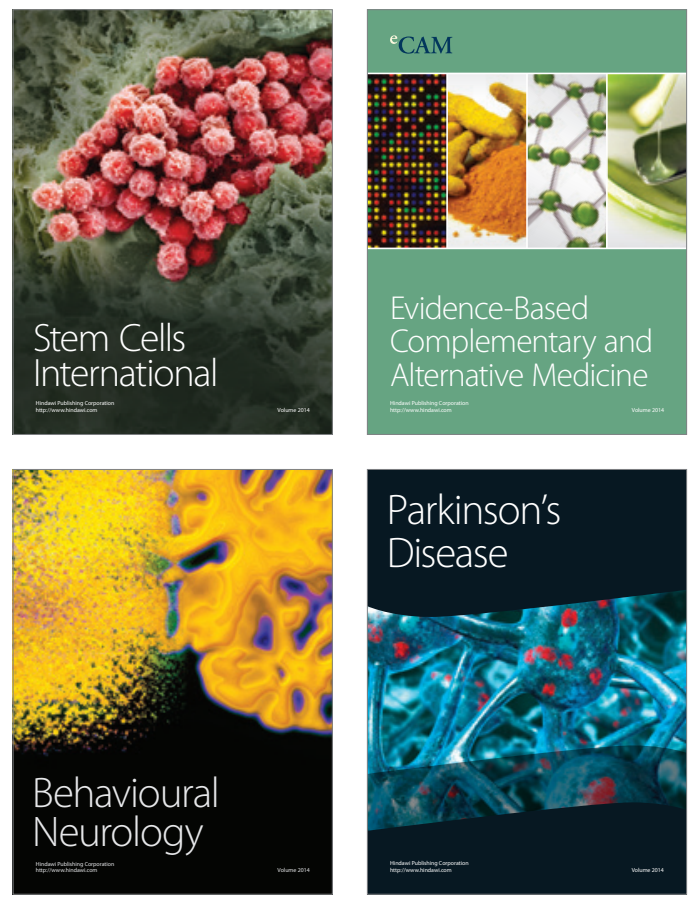
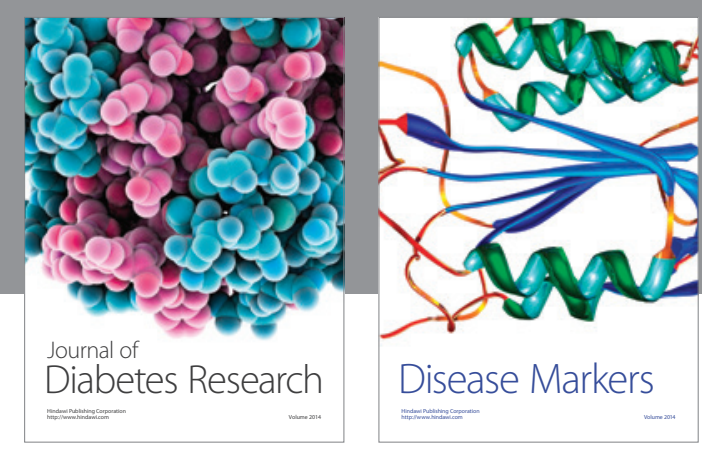

Disease Markers
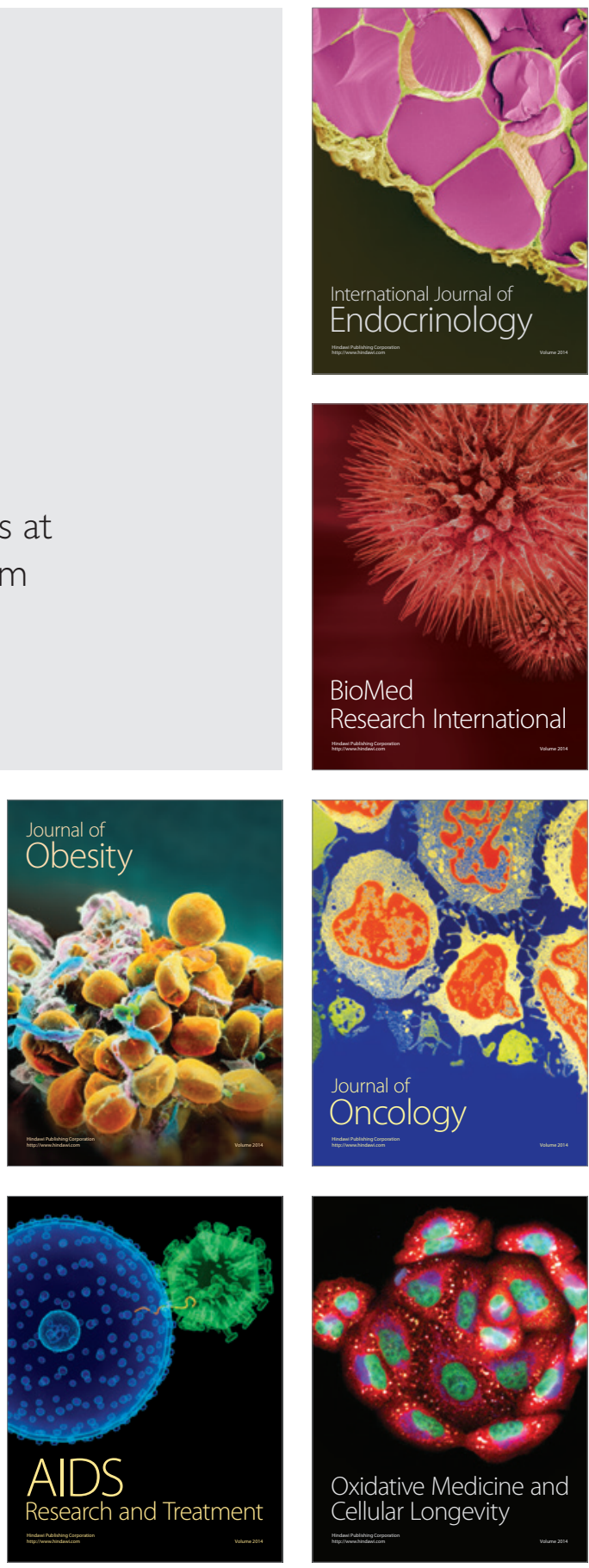